Observat $i$ on of the pol arizat $i$ on el ectric field in the int ense neutral ized prot on beam propagat ing undef I ectedly across a magnet i c $\mathrm{fi}$ el d

\begin{tabular}{|l|l|}
\hline 著者 & $\begin{array}{l}\text { I kehat a T., Kanada Kei i chi , I shi zuka Hi roshi , } \\
\text { Kawabe T. , M yoshi Syoi chi }\end{array}$ \\
\hline $\begin{array}{l}\text { j our nal or } \\
\text { publ i cat i on ti t l e }\end{array}$ & Physi cal Revi ew A \\
\hline vol une & 25 \\
\hline number & 6 \\
\hline page r ange & $3415-3418$ \\
\hline year & $1982-01-01$ \\
\hline URL & ht t p: //hdl . handl e. net /2297/14415 \\
\hline
\end{tabular}




\title{
Observation of the polarization electric field in the intense neutralized proton beam propagating undeflectedly across a magnetic field
}

\author{
T. Ikehata, K. Kamada, ${ }^{*}$ H. Ishizuka, ${ }^{\dagger}$ T. Kawabe, and S. Miyoshi \\ Institute of Physics, University of Tsukuba, Ibaraki 305, Japan
}

(Received 25 September 1981)

\begin{abstract}
A neutralized proton beam (averaged velocity $\bar{v}=3.6 \times 10^{8} \mathrm{~cm} / \mathrm{s}$, velocity spread $\Delta v=0.9 \times 10^{8} \mathrm{~cm} / \mathrm{s}$, proton current density $\left.\sim 18 \mathrm{~A} / \mathrm{cm}^{2}\right)$ is injected into a transverse magnetic field $B_{4}(\leq 3 \mathrm{kG})$. It is found that the electric field which satisfies the condition $E=\bar{v} B_{\perp}$ value is formed in the beam and the beam propagates across the magnetic field by the polarization drift when the dielectric constant $\epsilon=1+\left(\omega_{p i} / \omega_{c i}\right)^{2}$ of the beam is larger than $100-180$. The lowest value of $\epsilon$ for the beam propagation given above is consistent with the theoretical prediction $\epsilon \gg 43$.
\end{abstract}

The propagation of intense neutralized ion beams across a magnetic field is an important problem in their application to fusion research, such as the formation of reverse-field ion rings ${ }^{1}$ and the heating of magnetically confined plasmas. ${ }^{2}$

In general, intense neutralized ion beams are space charge and often current neutralized by electrons and take the form of plasma streams with large forward momentums. ${ }^{3}$

The mechanism of the cross-field propagation of these beams is the polarization drift of the ions and the electrons by the electric field induced in the beam. This electric field is produced as a result of the polarization derived by the Lorentz force perpendicular to both directions of the propagation of the beam and the magnetic field. An analytical model dealing with the cross-field propagation of the beam shows that the electric field which has the strength $E=v B_{\perp}$ and the direction opposite to the Lorentz force $V$ is necessary for the undeflected propagation of the beam across the magnetic field. Furthermore, this electric field is formed automatically in the beam when the dielectric constant of the beam

$$
\epsilon=1+\left(\omega_{p i} / \omega_{c i}\right)^{2} \gg\left(m_{i} / m_{e}\right)^{1 / 2},
$$

where $v$ is the velocity of the beam, $B_{\perp}$ the strength of the transverse magnetic field, $\omega_{p i}$ the ion plasma frequency of the beam, $\omega_{c i}$ the ion gyrofrequency, $m_{i}$ the ion mass, and $m_{e}$ is the electron mass. ${ }^{4}$

When the beam has the velocity spread of the ions, it is pointed out theoretically that $v$ must be re- placed by the averaged velocity $\bar{v}{ }^{5}$

A few experiments on the propagation of the beam across the magnetic field were reported previously, ${ }^{6,7}$ however, the observation of the polarization electric field and comparison of the results with the theoretical prediction described above with the velocity distribution of the ions taken into account are not reported.

In this paper, we report the formation of the polarization electric field by the propagation of the intense neutralized proton beam with the velocity spread across the magnetic field. In particular, the following two points are emphasized: Firstly, the polarization electric field in the beam and the velocity distribution of the protons are measured. The experimental results agreed with the theoretical prediction $E=\bar{v} B_{1}$. Secondly, the lowest value of $\epsilon$ which is necessary for the formation of the polarization electric field is determined experimentally, and it is compared with the theoretical prediction of satisfying the condition $\epsilon>>\left(m_{i} / m_{e}\right)^{1 / 2}$.

The apparatus used in the experiment is shown in Fig. 1. For convenience, we define the coordinates $x, y$, and $z$ as shown in the figure.

The neutralized proton beam is produced by a reflex triode ${ }^{8}$ which consists of an anode made of a 4- $\mu \mathrm{m}$ Mylar sheet and a cathode placed $2 \mathrm{~cm}$ apart from the anode. The anode is energized by a Marx generator. A magnetic field $B_{z}$ of $6 \mathrm{kG}$ is applied to the triode to eliminate electron losses to the anode support and to make the beam production reproducible. The applied voltage to the anode is fixed at $260 \mathrm{kV}$ throughout the experiment. 


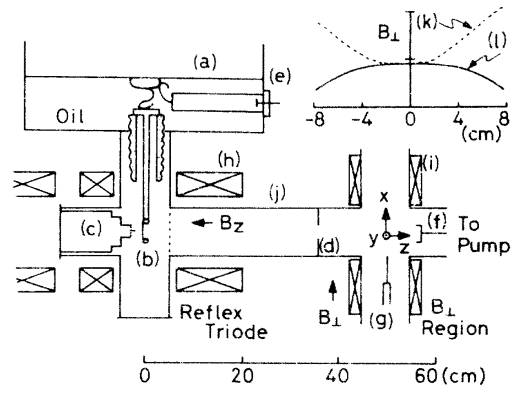

FIG. 1. Schematic diagram of the apparatus; (a) Marx generator, (b) anode, (c) cathode, (d) aperture, (e) voltage monitor, (f) biased charge collectors, $(\mathrm{g})$ a pair of Langmuir probes, (h) $B_{z}$ coils, (i) $B_{\perp}$ coils, (j) Pyrex tube, (k) profile of $B_{\perp}$ on the $x$ axis, and (1) profile of $B_{\perp}$ on the $z$ axis.

The beam extracted from the anode propagates about $50 \mathrm{~cm}$ downstream in the drift tube. The drift tube is a Pyrex tube and is evacuated down to $2 \times 10^{-5}$ Torr by oil diffusion pumps. The beam is collimated by an aperture of $2.5 \mathrm{~cm}$ in diameter placed in the drift tube at $35 \mathrm{~cm}$ downstream from the anode, in order for the beam not to come in contact with the wall.

The transverse magnetic field $B_{1}$, which has a strength of up to $3 \mathrm{kG}$ at the center of the $B_{\perp}$ region and a duration of $10 \mathrm{~ms}$, is produced by a pair of mirror coils energized by a capacitor bank. The strength of $B_{\perp}$ is constant, within $\sim 10 \%$, in a range $-4 \lesssim x \leqq 3 \mathrm{~cm}$ and $-5 \lesssim z \lesssim 5 \mathrm{~cm}$ as shown in Fig. 1. We represent the strength of $B_{\perp}$ at a point $x, y$, and $z$ as $B_{1}(x, y, z)$.

Radial profiles of the proton current density for $B_{1}=0$ were measured by biased charge collectors with a bias voltage of $-300 \mathrm{~V}$ and are shown in Fig. 2(a). It is found that the beam has a peak value of proton current density $\sim 18 \mathrm{~A} / \mathrm{cm}^{2}$, a pulse width of $200-300 \mathrm{~ns}$, a divergence angle $\sim 4^{\circ}$ and a circular cross section of $\sim 5 \mathrm{~cm}$ in di-
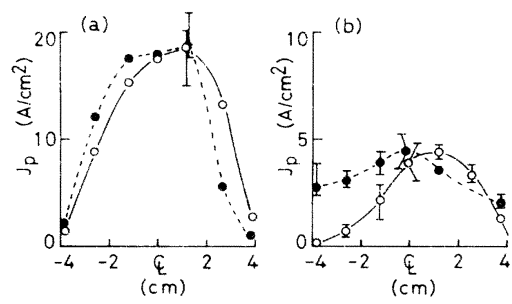

FIG. 2. Radial profiles of the proton current density $J_{p}$ of the beam measured (a) at $z=0$ for $B_{1}(0,0,0)=0$ and (b) at $z=6 \mathrm{~cm}$ for $B_{\perp}(0,0,0)=2 \mathrm{kG}$. Open (closed) circles show proton current density as a function of $y$ $(x)$. ameter at $z=0$. The total proton current of the beam is estimated to be $\sim 350 \mathrm{~A}$ from Fig. 2(a).

The net current density of the beam (the sum of the proton current density and the electron current density) was measured by the charge collector with a null bias voltage and was found to be $\sim 4 \mathrm{~A} / \mathrm{cm}^{2}$ at $x, y$, and $z=0$. From the facts that the beam propagates a distance $\sim 50 \mathrm{~cm}$ without the significant loss of the protons and that the net current density of $\sim 4 \mathrm{~A} / \mathrm{cm}^{2}$ is about $\frac{1}{5}$ of the proton current density of $\sim 18 \mathrm{~A} / \mathrm{cm}^{2}$, the beam is inferred to be space-charge neutralized and almost current neutralized.

The velocity distribution of the protons was measured by a $180^{\circ}$ deflection magnetic analyzer which was equipped with an entrance slit of $3 \mathrm{~mm}$ in width and 15 Faraday cups of $1 \mathrm{~cm}$ in diameter as detectors. It was found that the distribution was nearly a shifted Maxwellian with the averaged velocity of $\bar{v}=3.6 \times 10^{8} \mathrm{~cm} / \mathrm{s}$ and with the velocity spread of $\Delta v=0.9 \times 10^{8} \mathrm{~cm} / \mathrm{s}$ at the time when the proton current reached its peak. This distribution appears not to vary over the cross section of the beam because proton current pulses observed at different radii begin to rise and reach their peaks at the same time.

Radial profiles of the proton current density of the beam in the transverse magnetic field were measured at $z=6 \mathrm{~cm}$ for $B_{1}(0,0,0)=2 \mathrm{kG}$ and are shown in Fig. 2(b). If only the Lorentz force acts on the protons, the beam will be deflected and the peak of the proton current density will shift to $y \sim 5 \mathrm{~cm}$ as a result of single-particle motions of the protons. However, the significant shift of the current peak is not observed in Fig. 2(b) and was not observed for $B_{\llcorner}(0,0,0)=3 \mathrm{kG}$ either. The reason for this may be that the electric field is formed in the beam along the $y$ axis by the polarization, which makes the beam go straight across $B_{\perp}$ by the polarization drift.

A pair of Langmuir probes at floating potential arranged along the $y$ axis with a separation of 1.5 $\mathrm{cm}$ was inserted into the beam to measure the polarization electric field directly. The probe consists of a tungsten wire of $0.5 \mathrm{~mm}$ in diameter connected to a resistive voltage divider. The resistive divider consists of a series of ten resistors of $6 \mathrm{k} \Omega$ and a $50-\Omega$ termination resistor and is sealed in a Pyrex glass pipe. The use of the Pyrex glass pipe instead of a metal pipe as the container serves to increase the capacitive impedance of the probe input and therefore improves the frequency response of the probe. The input resistance $60 \mathrm{k} \Omega$ of the probe was found to be sufficient to measure the 
floating potential in the beam since the probe output reduced by only $\sim 10 \%$ when the resistance 60 $\mathrm{k} \Omega$ decreased to $10 \mathrm{k} \Omega$. The difference between the two probe outputs (the upper probe at $y=1.0$ $\mathrm{cm}$ and the lower probe at $y=-0.5 \mathrm{~cm}$ ) is displayed on an oscilloscope. The strength of the electric field along the $y$ axis is evaluated from the potential difference between the two probes and the probe separation of $1.5 \mathrm{~cm}$. It was verified that the potential difference between the two probes was proportional to the probe separation and was almost null when the probes were arranged along the $z$ axis.

Typical oscillograms of the electric field, together with the oscillogram of the proton current pulse, are shown in Fig. 3(a). The strength of the electric field is proportional to the strength of $B_{\perp}$ ([2] and [3]) and is null for $B_{1}=0$ ([1]). The polarity of the electric field reverses by reversing the polarity of $B_{1}$, but its amplitude does not change ([4]). We adopt as the typical value of the electric field, the one at the time when the proton current reaches its peak, after $200 \mathrm{~ns}$ from its rise ([5]).

The measured electric field $E$ (open circles) as a function of $B_{\perp}$ is shown in Fig. 3(b), where the solid line represents the polarization electric field $E=\bar{v} B_{\perp}$ calculated from $\bar{v}=3.6 \times 10^{8} \mathrm{~cm} / \mathrm{s}$. Measured values agree well with the theoretical prediction. Therefore, it can be concluded that the necessary and sufficient electric field for the undeflected propagation of the beam with the velocity spread which is expressed as $E=\bar{v} B_{\perp}$ is formed in the beam. Furthermore, the electric field was measured when a conducting plate was placed in
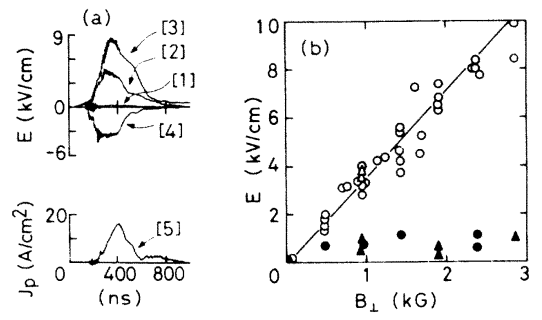

FIG. 3. (a) Typical oscillograms of the electric field in the beam for $B_{1}(0,0,0)=0$ [1], $1 \mathrm{kG}$ [2], $2 \mathrm{kG}$ [3], and $1 \mathrm{kG}$ of the reversed polarity [4], and of the proton current pulse [5]. Data are obtained from different shots and at $x=z=0$. (b) Electric field $E$ along the $y$ axis as a function of the strength of $B_{\perp}$ (open circles and triangles). Electric fields observed when a conducting plate is placed at $x=2.5 \mathrm{~cm}$ are also shown (closed circles and triangles). Circles (triangles) are data points for $B_{\perp}$ of the positive (negative) $x$ polarity. The solid line represents the theoretical prediction $E=\bar{v} B_{1}$. the beam at $x=2.5 \mathrm{~cm}$ with its surface parallel to the beam and perpendicular to $B_{\perp}$ field lines. The results (closed circles) are also shown in Fig. 3(b). In this case, the electric field is almost null regardless of the strength of $B_{\perp}$. This means that the electric field is shorted out by the conducting plate.

The distribution of the electric field in the beam along $B_{\perp}$ field lines was measured by moving the probes along the $x$ axis. The results are shown in Fig. $4(\mathrm{a})$ for $B_{1}(0,0,0)=1$ and $2 \mathrm{kG}$, where the solid lines represent distributions of the electric field $E=\bar{v} B_{\perp}$ calculated from $\bar{v}=3.6 \times 10^{8} \mathrm{~cm} / \mathrm{s}$ and from the profile of $B_{\perp}$ on the $x$ axis shown in Fig. 1. This figure presents the following features as to the polarization of the beam. Firstly, the electric field, which satisfies the condition $E=\bar{v} B_{\perp}$ locally in accordance with the spacial variation in $B_{\perp}$ is formed automatically in the beam at $-5 \lesssim x \leqslant 5 \mathrm{~cm}$. Secondly, the electric field decreases rapidly at $x \geq 5 \mathrm{~cm}$ in spite of the increase in $B_{\perp}$ and is far below that necessary for the polarization propagation of the beam. Considering that the number density of the protons at $x \geq 5 \mathrm{~cm}$ is
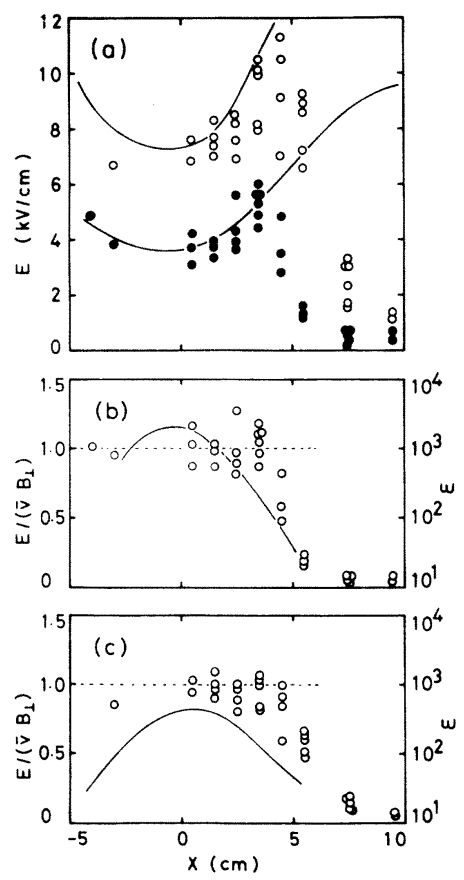

FIG. 4. (a) Distribution of the electric field $E$ in the beam on the $x$ axis for $B_{1}(0,0,0)=1 \mathrm{kG}$ (closed circles) and $2 \mathrm{kG}$ (open circles). Solid lines are from $E=\bar{v} B_{1}$. (b) Comparison between distributions on the $x$ axis of the normalized electric field $E /\left(\bar{v} B_{\perp}\right)$ (open circles) and of the value of $\epsilon$ of the beam (the solid line) for $B_{1}(0,0,0)=1 \mathrm{kG}$ and $(\mathrm{c})$ for $B_{1}(0,0,0)=2 \mathrm{kG}$. 
$\lesssim \frac{1}{5}$ of the peak value, the reason for the reduction in the electric field at $x \geq 5 \mathrm{~cm}$ is that the number density of the protons is too small to form the full electric field $E=\bar{v} B_{\perp}$ by the polarization. Therefore, we can determine experimentally the lower bound of the value of $\epsilon=1+\left(\omega_{p i} / \omega_{c i}\right)^{2}$ of the beam, necessary for the formation of the polarization electric field, by the evaluation of $\epsilon$ at the transition point where the electric field $E$ decreases rapidly from the full value $\bar{v} B_{1}$.

The distribution on the $x$ axis of the normalized electric field $E /\left(\bar{v} B_{1}\right)$ calculated from data shown in Fig. 4(a) and the distribution on the $x$ axis of the value of $\epsilon V$ are shown in Figs. 4(b) and 4(c) for $B_{\perp}(0,0,0)=1$ and $2 \mathrm{kG}$, respectively. The dielectric constant $\epsilon$ is evaluated from the proton current density, the averaged velocity of the protons $\bar{v}=3.6 \times 10^{8} \mathrm{~cm} / \mathrm{s}$, and the profile of $B_{1}$ shown in Fig. 1. The normalized electric field has the value $\sim 1.0$ and is constant at $-4 \lesssim x \lesssim 4 \mathrm{~cm}(-3 \leqq x \leqq 3$ $\mathrm{cm})$ for $B_{1}(0,0,0)=1 \mathrm{kG}(2 \mathrm{kG})$ in spite of the wide variation in $\epsilon$ in a range $100-2000(180-450)$. The transition of the value of $E /\left(\bar{v} B_{1}\right)$ from $\sim 1.0$ to $\ll 1.0$ occurs at $x=4 \mathrm{~cm}(3-4 \mathrm{~cm})$ where the value of $\epsilon$ is $100(100-180)$. This value of $\epsilon$ in a range $100-180$ is consistent with the theoretical prediction $\epsilon \gg\left(m_{i} / m_{e}\right)^{1 / 2}=43$ for protons.

In summary, the neutralized proton beam $\left(\bar{v}=3.6 \times 10^{8} \mathrm{~cm} / \mathrm{s}\right.$, which is equivalent to $70 \mathrm{keV}$ in energy, $\Delta v=0.9 \times 10^{8} \mathrm{~cm} / \mathrm{s}, \sim 18 \mathrm{~A} / \mathrm{cm}^{2}$ and $200-300 \mathrm{~ns}$ ) is injected into the transverse magnetic field of up to $3 \mathrm{kG}$. The polarization electric field necessary for the undeflected propagation of the beam is observed. It is found that this electric field is formed so as to satisfy the condition $E=\bar{v} B_{\perp}$ locally in the beam in accordance with the spacial variation in $B_{\perp}$ as far as the dielectric constant $\epsilon$ is sufficiently large. The lowest value of $\epsilon$ necessary for the formation of the polarization electric field is derived from the experiment to be $100-180$, which is consistent with the theoretical prediction $\epsilon>>\left(m_{i} / m_{e}\right)^{1 / 2}=43$ for protons.
*Present address: Department of Physics, Kanazawa University, Kanazawa 920, Japan.

${ }^{\dagger}$ Present address: Department of Physics, University of California, Irvine, California 92717.

${ }^{1}$ H. H. Fleishmann and T. Kammash, Nucl. Fusion 15, 1143 (1975).

${ }^{2}$ E. Ott and W. M. Manheimer, Nucl. Fusion 17, 1057 (1977).

${ }^{3}$ S. Humphries, Jr., Nucl. Fusion 20, 1549 (1980).
${ }^{4}$ W. Peter and N. Rostoker, Bull. Am. Phys. Soc. 25, 1007 (1980).

${ }^{5}$ G. O. Barney, Phys. Fluids 12, 2429 (1969).

${ }^{6}$ K. Kamada, C. Okada, T. Ikehata, H. Ishizuka, and S. Miyoshi, J. Phys. Soc. Jpn. 4ㅌ, 1963 (1979).

${ }^{7}$ S. Robertson, H. Ishizuka, W. Peter, and N. Rostoker, Phys. Rev. Lett. 47, 508 (1981).

${ }^{8}$ D. S. Prono, J. W. Shearer, and R. J. Briggs, Phys. Rev. Lett. 37, 21 (1976). 